\title{
The flip-flap puzzle flap: Another recycling option
}

\author{
Silvia Gandolfi ${ }^{1}$, Raphael Carloni ${ }^{2}$, Matthieu Gilleron ${ }^{3}$, Albane Bonmarchand ${ }^{1}$, \\ Isabelle Auquit-Auckbur ${ }^{1}$ \\ ${ }^{1}$ Department of Plastic, Reconstructive and Hand Surgery, Charles Nicolle University Hospital, Rouen; ${ }^{2}$ Department of Plastic Surgery, \\ Hôpital Privé de l'Estuaire, Le Havre; ${ }^{3}$ Department of Orthopedic Surgery, Charles Nicolle University Hospital, Rouen, France
}

Post-traumatic soft tissue defects sometimes require sequential flap coverage to achieve complete healing. In the era of propeller flaps, which were developed to reduce donor site morbidity, Feng et al. introduced the concept of the free-style puzzle flap, in which a previously harvested flap becomes its own donor site by recycling the perforator. However, when a perforator cannot be found with a Doppler device, we suggest performing a new type of flap, the flip-flap puzzle flap, which combines two concepts: the free-style puzzle flap and the flip-flap flap described by Voche et al. in the 1990s. We present the cases of three patients who achieved complete healing through this procedure.

Keywords Surgical flaps / Perforator flaps / Reconstructive surgical procedures / Extremities / Soft tissue injuries
Correspondence: Raphael Carloni Department of Plastic Surgery, Hôpital Privé de l'Estuaire, 505 rue Irène Joliot Curie, Le Havre 76620, France Tel: +33-2-76-89-94-32 E-mail: raphaelcarloni@hotmail.com

Received: 23 Aug $2018 \bullet$ Revised: 22 Nov $2018 \bullet$ Accepted: 8 Dec 2018

pISSN: 2234-6163 • elSSN: 2234-6171 • https://doi.org/10.5999/aps.2018.00983• Arch Plast Surg 2019;46:176-180

\section{INTRODUCTION}

In recent years, plastic surgeons have focused their attention on propeller flaps to provide a customized reconstruction solution with minor morbidity of the donor site. If loss of substance recurs, sequential flap coverage may be required, but in the era of propeller flaps, the recent concept of the free-style puzzle flap, introduced in 2013 by Feng et al. [1], could be an excellent solution. In this technique for "recycling" a flap, a previously harvested flap becomes its own donor site by reusing a perforator to overcome difficulties in reconstruction.

This concept has revolutionized approaches to reconstruction techniques, which are becoming less and less invasive in terms of donor site morbidity and the duration of both surgery itself and the postsurgical stay.

However, if conventional flaps repeatedly fail, a propeller puzzle flap is not always indicated. When a perforator cannot be found with a Doppler device on the previously-performed flap, surgeons cannot perform a puzzle flap, and may still be obliged to choose among sequential and conventional local or free flaps. In addition, in cases of multiple flap failures, patients desire a rapid and minimally invasive surgical procedure.

To overcome the issue of the absence of a perforator on a previously-performed flap, and to avoid invasive additional surgical strategies, we developed a new type of flap, the flip-flap puzzle flap, which combines two concepts: the free-style puzzle flap and the flip-flap flap described by Voche et al. in the 1990s [2,3], which we routinely use this technique in our unit to cover the dorsal phalanges in hand surgery.

\section{IDEA}

The flip-flap puzzle flap is a simple de-epidermized turned flap harvested from a previously-performed flap. The principle of the technique is simple, as the goal is to harvest a de-epidermized and turned flap from a previously-performed vital flap and 


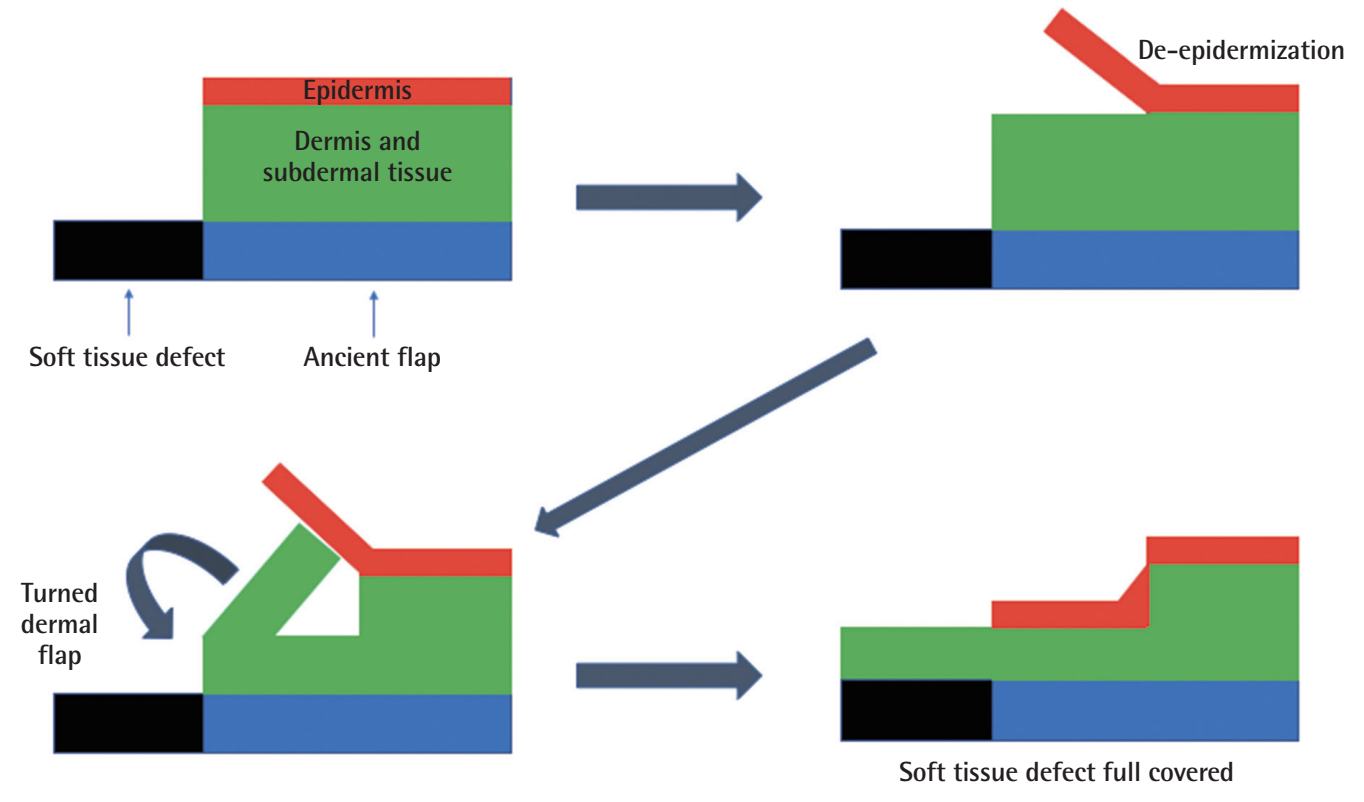

for use in cases with recurrent loss of substance (Fig. 1). Its boundary lines are marked on the original flap, according to the limits of the recurrent defect. Then, we de-epidermize the flipflap flap in the original flap with a scalpel and make an incision incorporating the dermis and subdermal tissue on three of its edges to raise a dermal turned flap. The dermis and vascular network of the original flap are then turned $180^{\circ}$ and positioned on the soft tissue defect. The de-epidermized skin can then be re-positioned in its original position (Supplemental Video 1).

This kind of flap can be performed as soon as 10 days after raising the first flap, and the 10-day interval must be respected in order to ensure subdermal neovascularization. Using this procedure, we were able to resolve three difficult cases of sequential flap failure. Patients were recruited with the approval of the Institutional Review Board (IRB No. 1902C), and informed consent was obtained according to the Declaration of Helsinki.

\section{Case 1}

A 70-year-old patient presented to Plastic and Hand Surgery Department at the Charles Nicolle University Hospital in Rouen, France with an avulsion injury of the forearm and the right hand. The soft tissue defect of the anterior side of the wrist and the forearm was covered with an interosseous posterior flap distally, and with a dermal regeneration matrix with non-expanded skin graft proximally. Partial necrosis of the skin graft occurred in the postoperative period, exposing tendons in the anterior forearm. For this reason, we applied a radial collateral artery perforator-based propeller flap, the distal part of which failed (Fig. 2A). The soft tissue defect secondary to this distal necrosis measured approximately $5 \times 3 \mathrm{~cm}$ and was covered by a flip-flap puzzle flap (Fig. 2B).

\section{Case 2}

A 56-year-old patient was initially admitted for a tibial pilon fracture and bimalleolar fracture of the right leg. He developed septic pseudarthrosis that required debridement of the infected site and a Masquelet procedure for his bone defect. Due to the lack of local laxity at the ankle level to cover the fracture focus and the cement, we performed a propeller flap using an anterior tibial artery perforator. After partial failure of this flap, sequential coverage by conventional flaps was performed (in order: a sural neurocutaneous flap that developed partial necrosis in its distal part, a hallucis brevis muscle flap that totally failed, and an internal plantar flap that developed partial necrosis in its distal part) (Fig. 3A). After the fourth flap, a recurrent defect of about $3 \times 5$ $\mathrm{cm}$ with cement exposure appeared next to the proximal part of the vital sural neurocutaneous flap that had been performed three months before. We performed a flip-flap puzzle flap raised on the sural neurocutaneous flap (Fig. 3B and C).

\section{Case 3}

A paraplegic 58-year-old woman was admitted to our Plastic Surgery Unit due to skin necrosis exposing the tibiotalar joint of the right ankle. We performed a propeller flap based on the an- 


\section{Fig. 2. Flip-flap puzzle flap for case 1}

(A) Figure A shows the preoperative view of the patient's forearm with a distal necrosis of a radial collateral artery perforator (RCAP)-based perforator flap. (B) Figure B shows the immediate postoperative view of a flip-flap puzzle flap harvested from the interosseous posterior flap (a, donor site on the interosseous posterior flap; $b$, flip-flap puzzle flap; c, RCAP-based propeller flap).
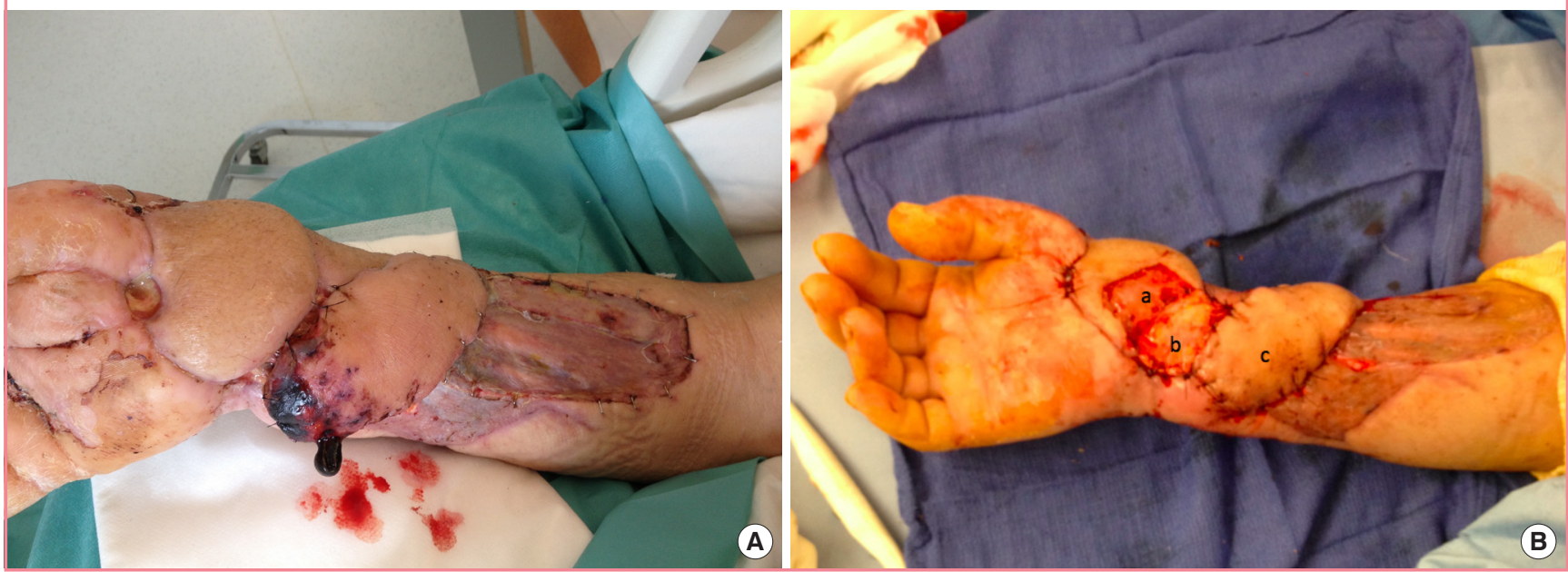

Fig. 3. Flip-flap puzzle flap for case 2

(A) Figure A shows the preoperative view of the patient's ankle (a, sural neurocutaneous flap; $b$, internal plantar flap with necrosis of its distal part). (B) Figure B shows the postoperative view at day 2 of the flip-flap puzzle flap harvested from the sural neurocutaneous flap (a, sural neurocutaneous flap; $b$, flip-flap puzzle flap covered with a skin graft; $c$, internal plantar flap). (C) Figure $\mathrm{C}$ shows the postoperative view at 1 month.
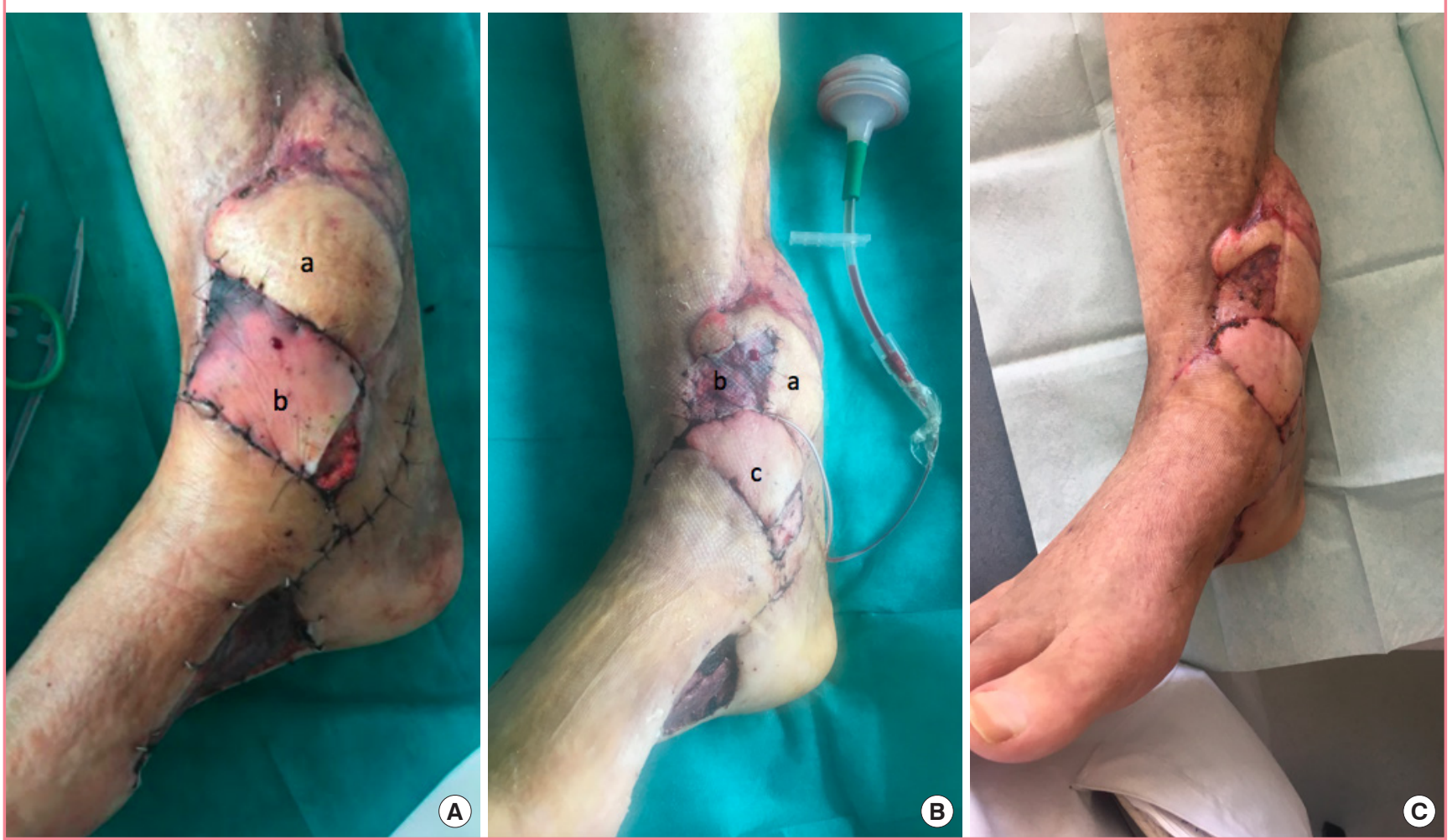

terior tibial artery. Superficial necrosis of the distal part of this flap, not exposing major anatomical structures, was observed. After necrosectomy, a skin graft was sufficient to fully cover the area. At 3 weeks, skin graft retraction exposed the talus bone
(Fig. 4A). The area of bone exposure measured $1 \times 2 \mathrm{~cm}$, and required a flip-flap puzzle flap to be raised on the tibial artery perforator flap (Fig. 4B). 


\section{Fig. 4. Flip-flap puzzle flap for case 3}

(A) Figure A shows the preoperative view of the patient's ankle $(a$, anterior tibial artery propeller flap; $b$. distal necrosis of the flap exposing the talus bone). (B) Figure $B$ shows the postoperative view at day 7 of the flip-flap puzzle flap harvested from the anterior tibial artery propeller flap $(a$, anterior tibial artery propeller flap; $b$, flip-flap puzzle flap covered with a skin graft).
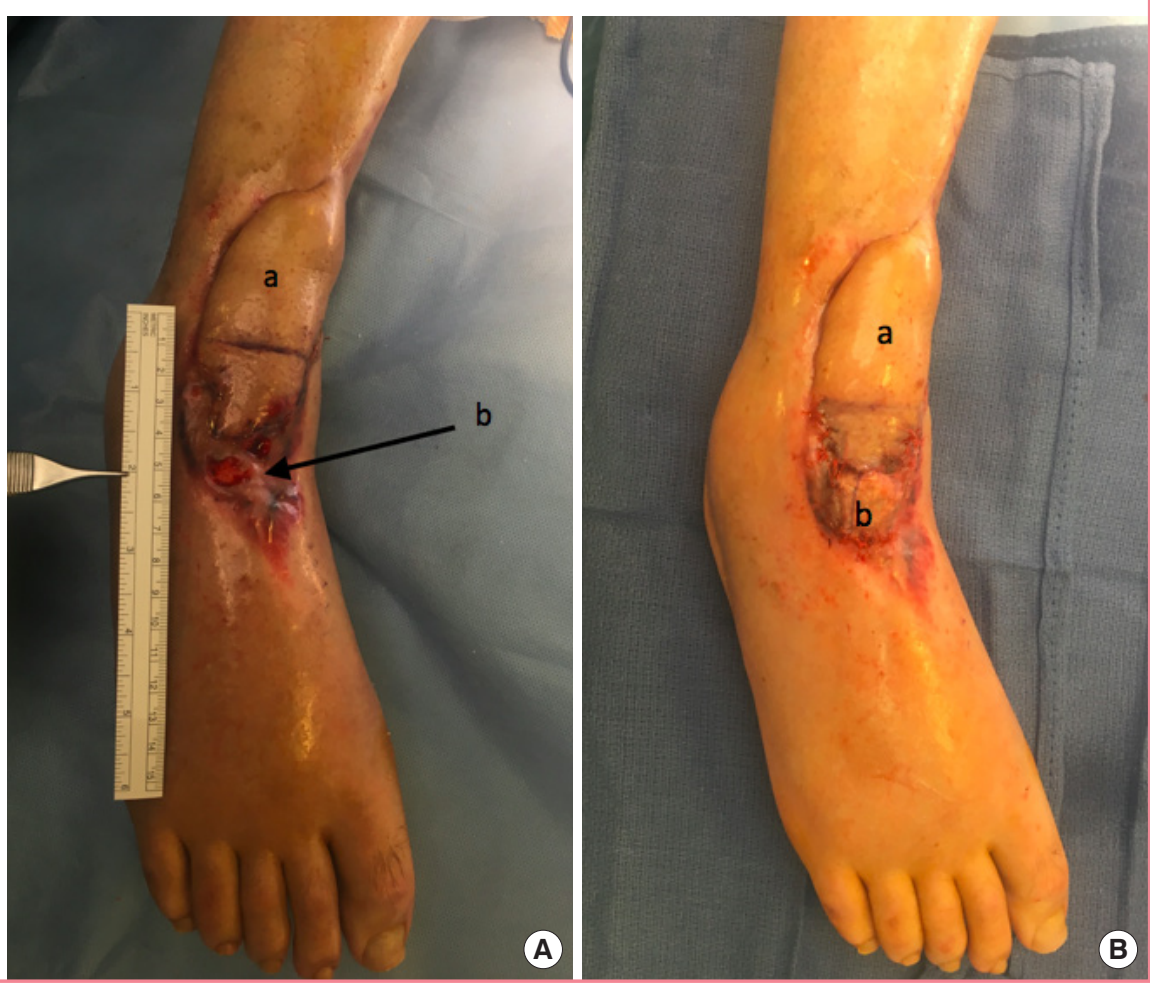

\section{DISCUSSION}

Herein, we report three cases of sequential flap failure that we were able to resolve through a simple de-epidermized turned flap harvested from a previously-performed flap. In the first case, after necrosis of the propeller flap, other options included free transfer, which would have been risky with a single arterial axis (we had ligated the ulnar artery in emergency surgery), or a MacGregor flap, which the patient categorically refused. In the second case, we performed four flaps to widely cover the area of cement. Possible alternative surgical solutions included mobilizing the sural neurocutaneous flap, but we did not have sufficient malleable tissue to fully transfer it; an external supramalleolar flap, but we did not want to sacrifice another donor site on an ankle with multiple injuries; or a cross-leg flap, which the patient refused. In the last case, the fragile state of our patient did not allow us to perform a major surgical procedure.

In the cases described herein, we aimed to recycle already harvested and vital flaps in accordance with the principle of donor site economy, especially because the areas with recurrent loss of substance were located near previously-performed and vital flaps. We considered the possibility of harvesting a free-style propeller puzzle flap, but opted for a simpler solution. We therefore needed a minimally invasive flap, for which this procedure was suitable.
At 3 months, our three patients had fully recovered, and no recurrent exposure was observed. In the first case, intensive physical therapy and a flexor tenolysis procedure are planned to improve functional outcomes. A radial collateral artery perforator flap thinning technique could be the next step to ameliorate the cosmetic appearance of the forearm. In our second case, the full coverage of the ankle and the absence of cement exposure allowed the orthopedic surgeons to manage the bone infection and to perform the second step of the Masquelet procedure with no obstacles involving sequential soft tissue coverage. In the third case, the aim of the covera ge was to prevent osteo-articular infection resulting from talus bone exposure, and our surgical solution fully succeeded in avoiding this possibility.

The principle of using a de-epidermized and turned flap is clearly not new. The use of dermal flaps based on sub-dermal vascularization is largely routine, with examples including the famous flip-flap flap used in hand surgery, which allows coverage of a soft tissue defect in a dorsal phalangeal unit by harvesting subdermal tissue on the palmar side of the same unit $[2,3]$. So far, we are not aware of any cases reported in the literature involving the reuse of a flap already performed through this simple and effective technique.

This technique requires a delay of approximately 10 days for the sub-dermal vascularization to reorganize [4].

In the cases described in this report, we opted for a flip-flap 
puzzle flap instead of a conventional flap for the following reasons: (1) the size of the defect was small, not exceeding $5 \times 3$ $\mathrm{cm}$; (2) the recurrent soft tissue defect was located near a previously-existing and vital flap; (3) the patient was exhausted by multiple previous surgical procedures and did not want more donor site-related scars; and (4) we wanted to preserve the donor sites of other flaps by recycling already-harvested and vital flaps, following the principle of donor site economy, which is an important concept for reconstructive surgeons $[5,6]$. Through this procedure, we were able to successfully cover recurrent soft tissue defects that were relatively small, but we do not know whether this technique can be applied to losses of substance with much larger dimensions.

All reconstructive surgeons must have a plan B in mind when deciding to repair a soft tissue defect, because it is necessary to follow the precept that "the patient is his own source of recurrence" [7]. Puzzle flaps provide another reconstructive solution without damaging other donor sites that could be used in the next step.

This simple technique, which combines the new concept of the puzzle flap with the wide use of a dermal turned flap like the flip-flap flap used in hand surgery, can be a solution for cases where reconstruction is difficult, the solutions start to be exhausted, and the patient desires a definitive and minimally-invasive solution.

\section{NOTES}

\section{Conflict of interest}

No potential conflict of interest relevant to this article was reported.

\section{Ethical approval}

The study was approved by the Institutional Review Board of the Charles Nicolle University Hospital (IRB No. 1902C) and performed in accordance with the principles of the Declaration of Helsinki. Written informed consents were obtained.

\section{Patient consent}

The patients provided written informed consent for the publication and the use of their images.

\section{Author contribution}

Acquisition of the data, drafting of the manuscript: Gandolfi S. Acquisition and interpretation of the data, drafting of the manu- script: Carloni R. Critical review of the article: Gilleron M, Bonmarchand A. Review of the article and final approval of the version to be published: Auquit-Auckbur I.

\section{ORCID}

Silvia Gandolfi https://orcid.org/0000-0001-5720-1741

Raphael Carloni https://orcid.org/0000-0001-9475-9314

Matthieu Gilleron https://orcid.org/0000-0002-0674-6925

Albane Bonmarchand https://orcid.org/0000-0001-92729365

Isabelle Auquit-Auckbur https://orcid.org/0000-0003-14246397

\section{Supplementary material}

Supplemental Video 1. Technique for harvesting a flip-flap puzzle flap. A flip-flap puzzle flap was harvested from the distal part of an anterior tibial artery propeller flap to cover exposed talus bone. The de-epidermized skin of the original flap was placed back in its previous position.

Supplemental data can be found at:

https://doi.org/10.5999/aps.2018.00983.v001

\section{REFERENCES}

1. Feng KM, Hsieh CH, Jeng SF. Free-style puzzle flap: the concept of recycling a perforator flap. Plast Reconstr Surg 2013;131:258-63.

2. Voche P, Merle M. The homodigital subcutaneous flap for cover of dorsal finger defects. Br J Plast Surg 1994;47:435-9.

3. Voche P, Beustes-Stefanelli M. Lambeau sous-dermique homodigital palmaire retourné ou lambeau flip-flap. In: Le Nen D, Hu W, Laulan J, editors. Approche pratique de la couverture des pertes de substance cutanée de la main et des doigts. Paris: Springer; 2007. p. 161-5.

4. Tsur H, Daniller A, Strauch B. Neovascularization of skin flaps: route and timing. Plast Reconstr Surg 1980;66:85-90.

5. Chaput B, Eburdery H, Laguerre J, et al. Chimeric flap and free-style puzzle flap for extended coverage of the foot and ankle. Plast Reconstr Surg 2015;135:797e-799e.

6. Herlin C, Lievain L, Qassemyar Q, et al. Freestyle free perforator flaps for heel reconstruction. Ann Chir Plast Esthet 2013;58:283-9.

7. Maghrabi HE, Grolleau JL, Chavoin JP. Traitement chirurgical des escarres. Techniques chirurgicales - Chirurgie plastique reconstructrice et esthétique $2011 ; 6: 1-10$. 